\title{
Detection of Diabetic Retinopathy from Retinal Fundus Image using Wavelet based Image Segmentation
}

\author{
Rajalaxmi Sahoo \\ Dept. of Computer Science and Application \\ Sambalpur University \\ Odisha
}

\author{
Chandra Sekhar Panda \\ Dept. of Computer Science and Application \\ Sambalpur University \\ Odisha
}

\begin{abstract}
In case of Diabetic Retinopathy, retina is damaged because of fluid leaks from blood vessels into the retina. According to ophthalmologists, some basic features are there to recognize diabetic retinopathy, such as blood vessel area, exudates, hemorrhages, micro-aneurysms and texture. Presence of exudates within the macular region is a main hallmark of diabetic which identifies its detection with a high sensitivity. Hence, detection of exudates is an important diagnostic task that can be determined by means of morphological techniques for better result. Here image segmentation has done to detect Diabetic Retinopathy by extracting exudate from retinal fundus images. Here the final extracted exudates contained the boundaries of proper segmented regions which can identify the severity of disease. Using Wavelet Transform decomposition method and morphological operations the feature like exudate is possible to extract from retinal fundus image to detect Diabetic Retinopathy. Here 4 datasets have been taken such as STARE, DRIVE, DIARETDB1 and DIARETDB0 for extraction of exudate from retinal image in which the proposed method obtained an average value of accuracy at $95.72 \%$, sensitivity at $95.87 \%$ and specificity at $96.2 \%$ respectively.
\end{abstract}

\section{Keywords}

Diabetic Retinopathy, Wavelet Transform, Exudates, STARE, DRIVE, DIARETDB1 and DIARETDB0.

\section{INTRODUCTION}

Image processing involves changing the nature of an image in order to either improve its pictorial information for human interpretation or perform a suitable situation for automatic machine perception. The retinal fundus images are usually of low contrast, uneven-illumination and blur of the details due to complex imaging environment. The purpose of retinal image enhancement is to improve the contrast and highlight the image.DR can be broadly classified into two types PDR (Proliferative diabetic retinopathy) and NPDR (NonProliferative Diabetic Retinopathy). These phases can be described according to the presence of specific DR features [1].

NPDR: The intra-retinal micro-vascular abnormalities signify either development of new vessel or re-building of preexisting vessels through endothelial cell production in the retinal tissues. 'They may be seen the accentuate spot or bigger blotch hemorrhages with uneven boundaries all through the fundus as fire-formed (flame-shaped).

PDR: With the occurrence of additional retinal ischemia, such diabetic retinopathy occurs. For instance, the abnormal vessels of vascularization are unstable, the hemorrhaging hypothetically prompting serious loss of vision is interminable. The optic nerve can be harmed with the increased pressure.
A wholly automated approach involving fundus image analysis by computer aided system could provide an immediate classification of retinopathy without the need for specialist opinions. CAD systems processes digital images to highlight conspicuous sections through segmentation to clear identify the affected area. The goal of CAD systems is to detect the earliest signs of abnormality in patients that human professional cannot, as in Diabetic Retinopathy. Here the aim of the study is to develop a Computer Aided Diagnostic system (CAD) capable of supporting in medical decisions; improving the diagnosis quality and decreasing the workload of medical professionals within the background of diagnose diabetic retinopathy.

Automated detection of DR lesions like exudates,microaneurysms, hemorrhages and blood vessels in digital fundus image can contribute to the diagnosis and screening of this disease. Here 2 main steps have been involved such aspreprocessing of the fundus image and segmentation as well to extract features of DR under which various operations have performed. Using wavelet based segmentation methodology it made possible to extract exudate from retinal fundus image.

The Exudates are shown as spatially random yellowish or whitish patches of varying shapes, sizes and localities present in the retinal images. These are the main cause and visible sign of diabetic retinopathy which can be gradually increase to vision loss. Detection of Exudates by ophthalmologists is a difficult process as they have to spend a considerable time on manual analysis [2].

\section{METHODOLOGY}

The methodological analysis of the work "Detection of diabetic retinopathy from retinal fundus image using Wavelet based Image segmentation" has been represented pictorially as below. 


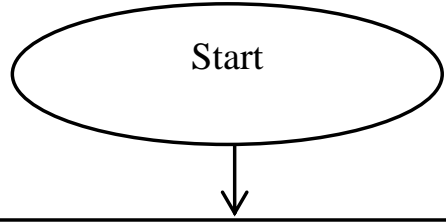

Input Retinal Fundus Image

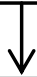

Pre-processing (Green Channel Extraction and Enhancement) Of input Image

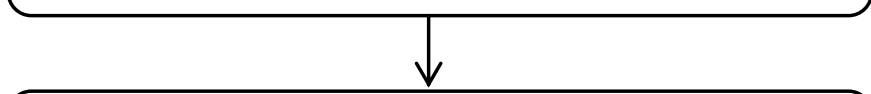

Image Segmentation using wavelet transformation and morphological operation

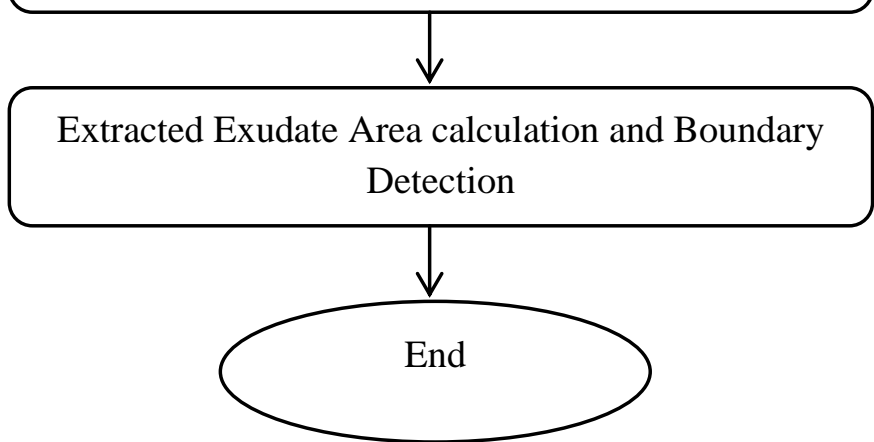

Fig.1 Flow chart for extraction of exudate from retinal fundus image to detect DR

\subsection{Pre-processing of an Image}

\section{Green-Channel Extraction:}

RGB representation is composed of red, green, and blue colors where in the green channel all minute details of image can be viewed. Using red channels only boundary is visible, and in blue channel image shows lots of noise. Due to these reasons green channel has used in the proposed system[3]. So the green channel among the RGB channels usually used for pre-processing.

Histogram Equalization for enhancement:

The purpose of image enhancement is to process an image such a way that it is more suitable than the original image for a specific application. It is quite acceptable that high contrast images have flat histograms to perform enhancement.

\subsection{Segmentation}

\section{Morphological Operation:}

According to fundamentals morphological image processing is like spatial filtering that the structuring element is moved across every pixel of the original image to give a pixel value in a new processed image and the value of this new pixel depends on the operation performed.

2D-Wavelet Transformation:

The Wavelet Transform of the input images is appropriately combined, and the new image is obtained by taking the inverse wavelet transform of the fused wavelet coefficients. Using Wavelet Transform the original image is decomposed at different scales using a pyramidal algorithm architecture which decomposition is along the vertical and horizontal directions and maintains the constant number of pixels required to describe the image.

\section{IMAGE SEGMENTATION USING WAVELET TRANSFORM}

Following subsections describe of image segmentation using wavelet transform

\subsection{Image Features Extraction}

Texture of the image can be characterized by the spatial distribution of grey levels in a neighbourhood. An image region has a constant texture. If a set of local properties of the texture region is constant, it is slowly changing or approximately periodic. Texture analysis is one of the most important techniques used in analysis. There are three primary issues in texture analysis: classification, segmentation and shape recovery from texture. Analysis of texture requires the identification of proper attributes or features that differentiate the textures of the image. Here texture segmentation is carried out by comparing co-occurrence matrix features Contrast and Energy of size $\mathrm{N} \times \mathrm{N}$ derived from discrete wavelet transform overlapping but adjacent sub images $\mathrm{Ci}$,j of size $4 \times 4$, both horizontally and vertically [4].

The algorithm of image features extraction involves

a) Decomposition, using one level Discrete Wavelet Transform, of each sub image $\mathrm{Ci}, \mathrm{j}$ of size $4 \times 4$ taken from the top left corner

b) Computation of the co-occurrence matrix features energy and contrast given in Eqs (1) and (2) from the detail coefficients, obtained from each sub image $\mathrm{Ci}, \mathrm{j}$

c) Forming new feature matrices

Energy $=\sum_{i, j=1}^{N} C_{i, j}^{2}$
Contrast $=\sum_{i, j=1}^{N}(i-j)^{2} C_{i, j}-------$

\subsection{Pixel Differences}

After the computation of co-occurrence matrix features, a new matrix with differences is obtained. It is carrying out by calculation the difference between the value by value of features both in horizontal and vertical directions. Then the segmentation band is formed across the texture boundaries.

\subsection{Boundary Detection}

The feature and information of the image can be obtained from the boundary of the image. Boundary extraction can help to implement the other process such as data extraction, corner detection or surface reconstruction. Boundary extraction process traces the exterior boundaries of object as well as boundaries of holes inside these objects.

\section{EXPERIMENTS AND RESULT}

The proposed technique has been analyzed on the openly accessible DRIVE (Digital Retinal Images for Vessel Extraction) [5], STARE (Structured Analysis of the Retina) [6], DIARETDB1 and DIARETDB0 databases that has been precisely marked by the experts. Here the dataset has included rich information about the vessel areas, the connected components of the fundus image. These images are of high resolution with a clear vision which can be used for further processing. The experiment has taken with four datasets under three conditions of Diabetic Retinopathy such as severe DR, mild DR and less DR according to the occupied area by exudates. 


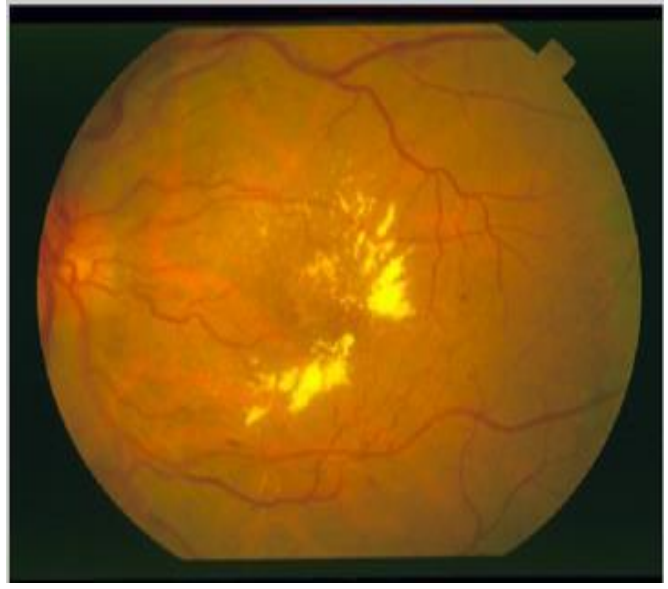

Fig.2 Original Retinal Fundus Image

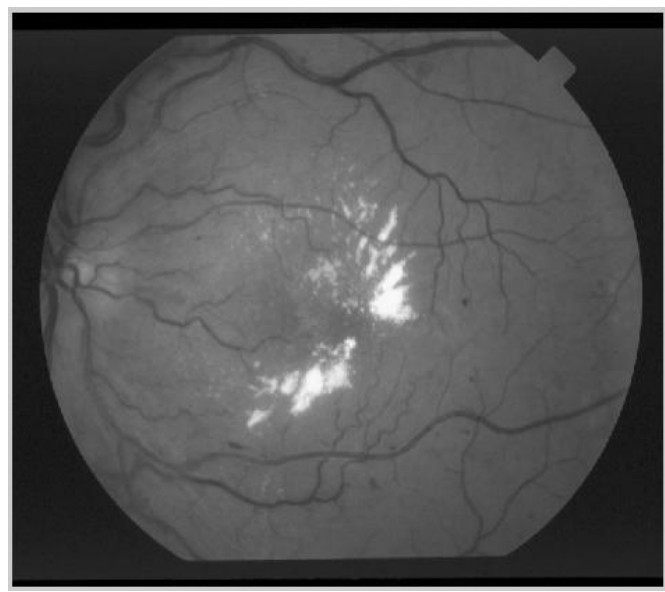

Fig.3 Green Channel Extracted Image

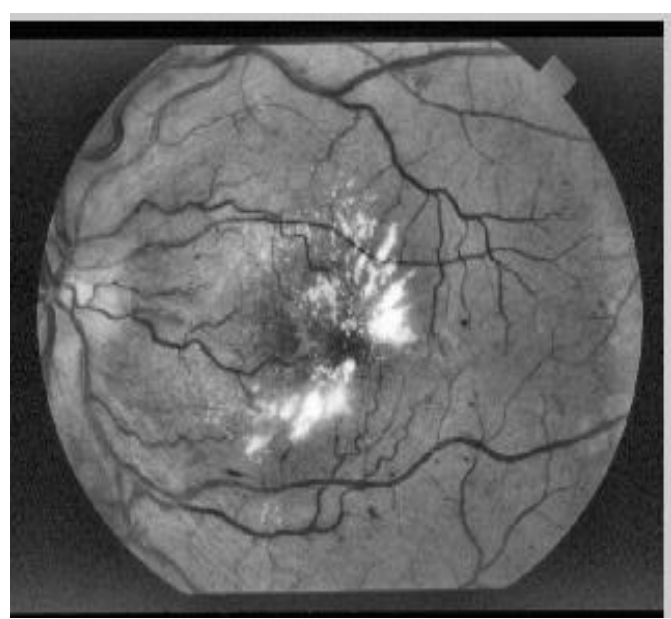

Fig.4 Histogram Equalized Enhanced Image

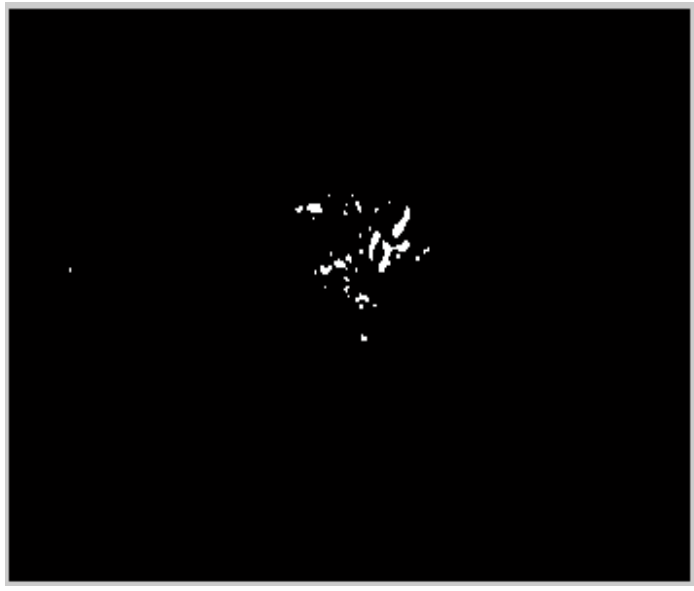

Fig.5 Morphological Binarized Image

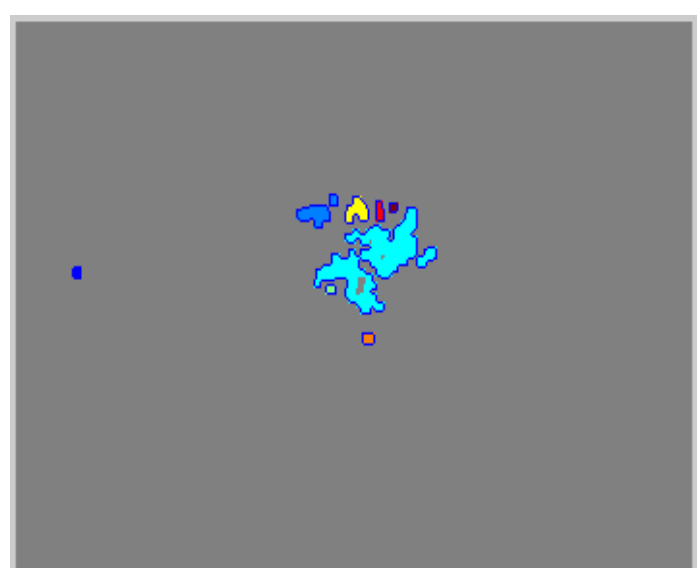

Fig.6 RGB Extraction of Exudate

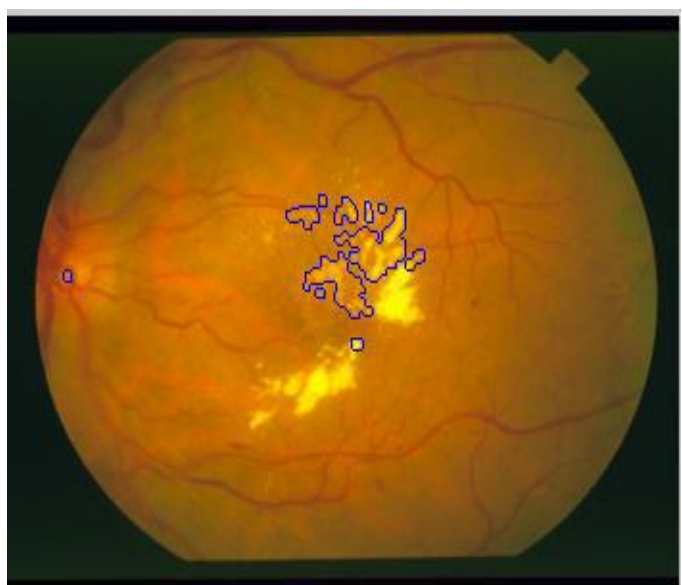

Fig.7 Boundary of Extracted Exudates

In the above experiment the retinal fundus images are gone through the proposed method in which first step is extraction of green channel from the original image then enhanced by histogram equalization method then according to algorithm with further proceedings using morphological operation and wavelet transformation the binary extraction of exudates has performed with some occupied area. Here the areas occupied by the exudates in corresponding retinal fundus images are being classified with some ranges which conclude the stages of DR. 
The sensitivity of the test indicates the probability that it would indicate a TP result when used on an infected subject. The sensitivity of the test could be determined by the formula

Sensitivity $=[\mathrm{TP} /(\mathrm{TP}+\mathrm{FN})]^{* 100}$

The specificity of a test measures the proportion of negatives, which are correctly identified that means the percentage of healthy people who are identified as not having the diabetic retinopathy. A theoretical prediction could achieve 100\% sensitivity (i.e. can predict all people from the diabetic retinopathy group as diabetic retinopathy) and 100\% specificity. The specificity of a test can produce a TN result when used on a non infected Diabetic Retinopathy. The specificity of a test could be determined by calculating:

Specificity $=[\mathrm{TN} /(\mathrm{TN}+\mathrm{FP})]^{*} 100$

The accuracy of the test indicates the closeness of the test that are taken in many sample images results to the true value and repeatability of the test. The accuracy of the test can be calculated by the formula:

Accuracy $=[(\mathrm{TP}+\mathrm{TN}) /(\mathrm{TP}+\mathrm{TN}+\mathrm{FP}+\mathrm{FN})] * 100$

Table1. Comparison of performance matrices on different datasets

\begin{tabular}{|l|c|c|c|}
\hline \multicolumn{1}{|c|}{$\begin{array}{c}\text { Standard } \\
\text { Database }\end{array}$} & $\begin{array}{c}\text { Sensitivity } \\
(\%)\end{array}$ & $\begin{array}{c}\text { Specificity } \\
(\%)\end{array}$ & Accuracy \\
& & & $(\%)$ \\
\hline DRIVE & $94.8 \%$ & $94.7 \%$ & $93.5 \%$ \\
\hline STARE & $94.1 \%$ & $95.9 \%$ & $95 \%$ \\
\hline DIARETDB1 & $97.7 \%$ & $96.6 \%$ & $97.1 \%$ \\
\hline DIARETDB0 & $96.9 \%$ & $97.6 \%$ & $97.3 \%$ \\
\hline
\end{tabular}

In the above measurements performance matrices, sensitivity, specificity and accuracy are calculated in such a way that the rate of exudate extraction can be maintained in a digitized format. The proposed CAD system gave a higher sensitivity rate of $96.9 \%$, specificity of $97.6 \%$ and accuracy of $97.3 \%$ respectively for DIARETDB0.

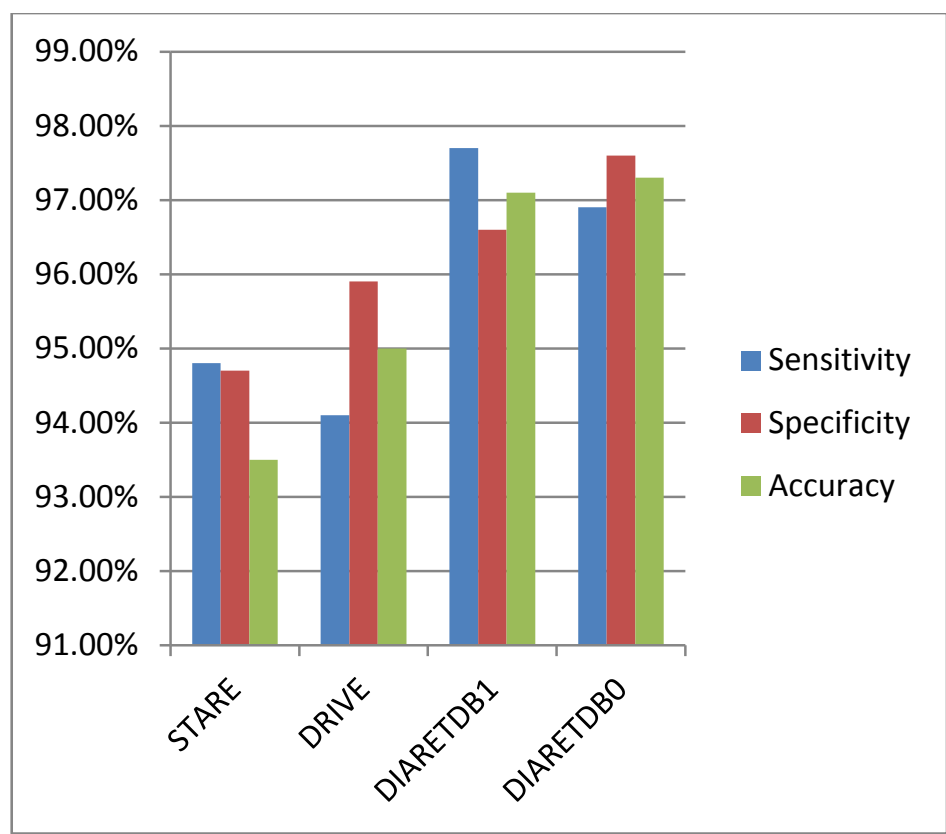

Fig.8 Performance Comparison of Different Datasets

Table2. Performance Comparison of Existing System with Proposed System

\begin{tabular}{|c|c|c|c|c|}
\hline $\begin{array}{l}\text { Standard } \\
\text { Database }\end{array}$ & $\begin{array}{l}\text { Comparisi } \\
\text { on } \\
\text { Methods }\end{array}$ & $\begin{array}{c}\text { Sensitivi } \\
\text { ty }(\%)\end{array}$ & $\begin{array}{c}\text { Specifici } \\
\text { ty } \\
(\%)\end{array}$ & $\begin{array}{c}\text { Accura } \\
\text { cy } \\
(\%)\end{array}$ \\
\hline $\begin{array}{l}\text { DRIVE, } \\
\text { STARE }\end{array}$ & $\begin{array}{c}\text { Kumar et } \\
\text { al(2012) } \\
{[7]}\end{array}$ & $97.1 \%$ & $98.3 \%$ & $95.7 \%$ \\
\hline $\begin{array}{c}\text { DRIVE,STA } \\
\text { RE, } \\
\text { DIARETDB1 }\end{array}$ & $\begin{array}{c}\text { Vimala et } \\
\text { al(2017) } \\
{[8]}\end{array}$ & $91.3 \%$ & $90.5 \%$ & $91.8 \%$ \\
\hline $\begin{array}{c}\text { DIARETDB0 } \\
, \\
\text { DRIVE, } \\
\text { STARE }\end{array}$ & $\begin{array}{c}\text { CVPR } \\
2009 \text { [9] }\end{array}$ & $95.7 \%$ & $94.2 \%$ & $97.1 \%$ \\
\hline $\begin{array}{c}\text { DRIVE, } \\
\text { STARE } \\
\text { DIARETDB1 } \\
, \\
\text { DIARETDB0 }\end{array}$ & $\begin{array}{c}\text { Rajput et } \\
\text { al(2015) } \\
{[10]}\end{array}$ & $\begin{array}{c}\text { Not } \\
\text { Specifie } \\
\mathrm{d}\end{array}$ & $\begin{array}{c}\text { Not } \\
\text { Specifie } \\
d\end{array}$ & $92 \%$ \\
\hline $\begin{array}{c}\text { DRIVE, } \\
\text { STARE } \\
\text { DIARETDB1 } \\
, \\
\text { DIARETDB0 }\end{array}$ & $\begin{array}{l}\text { Proposed } \\
\text { Method }\end{array}$ & $95.8 \%$ & $96.2 \%$ & $95.7 \%$ \\
\hline
\end{tabular}




\section{CONCLUSION}

Evaluation of the area and performance of the exudates detection has stated in this paper. The publicly available datasets STARE, DRIVE, DIARETDB0 and DIARETDB1 are used. The performance of the proposed exudates segmentation results are analyzed with respect to previously obtained results of different expert authors. Proposed method obtained an average value of accuracy $95.7 \%$, sensitivity of $95.8 \%$ and specificity of $96.2 \%$ respectively which resulting the active presence of disease in retina. Such study on diabetic retinopathy indicates the automated evaluation of digital retinal images could be used to screen for exudates. The results of this work can be evaluated to produce an automated system to detect exudates. Micro aneurysm and hemorrhage detection could be added to the system in order to increase its ability to verify the degree of diabetic retinopathy. It will be useful to extend this work by developing a system to detect such extra features.

\section{REFERENCES}

[1] Oliver Faust \& Rajendra Acharya U. \& E. Y. K. Ng \& Kwan-Hoong Ng \& Jasjit S. Suri. (2012) Algorithms for the Automated Detection of Diabetic Retinopathy Using Digital Fundus Images: A Review. J Med Syst 36: 145157.

[2] Sopharak A, B. Uyyanonvara and S. Barman (2009) Automatic exudate detection from non-dilated diabetic retinopathy retinal images using fuzzy $\mathrm{C}$ means clustering. Journal of Sensor, 9: 2148-2161.
[3] Zhu, X., Rangayyan, R.M., Ells, A.L.: Digital Image Processing for Ophthalmology: Detection of the Optic Nerve Head

[4] Joerg Meyer, Zhihe Zhang, Wavelet-based Image Segmentation Feature Recognition in Noise-Distorted Biomedical Images, University of California, Irvine, CA 92697-2625

[5] DRIVEdataset[Online]:http://www.isi.uu.nl/Research/Da tabases/DRIVE/

[6] STARE: Structured Analysis of the Retina, [Online]: http://www.ces.clemson.edul-ahoover/stare/

[7] Atul Kumara, Abhishek Kumar Gaurb, Manish Srivastavac ( 2012 ) A Segment based Technique for detecting Exudate from Retinal Fundus image, Procedia Technology 6: $1-9$

[8] Annie Grace Vimala, Kaja Mohideen (2017) Diabetic retinopathy screening system: A validation analysis with multiple fundus image databases, Biomedical Research; 28 (4): 1598-1603

[9] Automated Feature Extraction for Early Detection of Diabetic Retinopathy in Fundus Images, CVPR 2009 Submission

[10] Yogesh M, Rajput,Ramesh R, Manza,Manjiri B Patwari (2015) Extraction of Cotton Wool Spot using Multi Resolution Analysis and Classification using K-Means Clustering, International Journal of Computer Applications (0975 - 8887),National conference on Digital Image and Signal Processing, DISP 